\title{
A Geometric View on Quantum Tensor Networks
}

\author{
Alexander Tsirulev ${ }^{1, \star}$ \\ ${ }^{1}$ Faculty of Mathematics, Tver State University, Sadovyi per. 35, 170002 Tver, Russia
}

\begin{abstract}
Tensor network states and algorithms play a key role in understanding the structure of complex quantum systems and their entanglement properties. This report is devoted to the problem of the construction of an arbitrary quantum state using the differential geometric scheme of covariant series in Riemann normal coordinates. The building blocks of the scheme are polynomials in the Pauli operators with the coefficients specified by the curvature, torsion, and their covariant derivatives on some base manifold. The problem of measuring the entanglement of multipartite mixed states is shortly discussed.
\end{abstract}

\section{Introduction}

The quantum tensor networks, which will be considered below in a wide sense, give us a useful method for the approximation and high-performance processing of quantum states [1-3]. Since a real quantum computer should have a large number of qubits, say, $n \geq 1000$, the number of basis states is $2^{n}>10^{300}$. It means that the number of elementary unitary operations needed to input user (big) data in a quantum register should in general be of the same order of magnitude. For this reason, a decomposition of the density matrix of a state of such a system into a finite family of contracted tensors (tensor train) can be efficiently implemented only for some special classes of quantum states. On the other hand, geometric ideas and geometric tools, including the geometry of quantum tensor networks [4], are quite common in quantum computing and quantum information theory, especially in connection with the study of the entanglement [5,6] and the emergence of gravity [7]. This short report presents an outline of a new geometric approach to the simulation of quantum states by using quantum tensor networks with a relatively small number of independent parameters. This approach is based on covariant series in normal coordinates on the direct product of $k(k \ll n)$ four-dimensional manifolds equipped with suitable linear connections and the corresponding curvatures and/or torsions; we will consider only the case $k=1$, but the generalization to an arbitrary $k>1$ is obvious. Given a connection (or a (pseudo) Riemannian metric), one computes the covariant derivatives of the curvature and the torsion, and then computes coefficients of a quantum state as components of some tensor of rank $n$. Explicit formulas and recipes for coefficients of the series are given in Refs. [8-11].

Sec. 2 contains some necessary mathematical preliminaries and a short description of quantum states in the Pauli basis. In Sec. 3 the state space of a $n$-qubit quantum system is modelled by a fourdimensional manifold; we give a detailed description of the covariant expansions for the case of a linear connection with zero curvature and nonzero torsion. Sec. 4 presents an illustrative example of modelling the Greenberger-Horne-Zeilinger (GHZ) state for a quantum system of three qubits.

^e-mail: tsirulev.an@tversu.ru 


\section{Quantum states in the Pauli basis}

Let $\mathcal{V}_{n}=\mathcal{V}^{\otimes n}$ be the Hilbert space of a quantum system of $n$ distinguishable qubits, $\operatorname{dim}_{\mathbb{C}} \mathcal{V}=2$. We will assume that the space $\operatorname{End}_{\mathbb{C}} \mathcal{V}_{n}$ of endomorphisms of $\mathcal{V}_{n}$ is equipped with the natural Hermitian inner product, $\langle\hat{A}, \hat{B}\rangle=\operatorname{tr}\left(\hat{A}^{\dagger} \hat{B}\right)$. The Pauli basis in End $\mathbb{C}_{n} \mathcal{V}_{n}$ is a Hermitian, unitary, and orthogonal basis consisting of the $2^{2 n}$ elements

$$
\left\{\hat{\sigma}_{i_{1} \ldots i_{n}}\right\}_{i_{1}, \ldots, i_{n} \in\{0,1,2,3\}}, \quad \hat{\sigma}_{i_{1} \ldots i_{n}}=\hat{\sigma}_{i_{1}} \otimes \ldots \otimes \hat{\sigma}_{i_{n}},
$$

where $\hat{\sigma}_{i}$ are the single-qubit Pauli operators which have, in some fixed basis $\{|0\rangle,|1\rangle\} \in \mathcal{V}$, the form

$$
\hat{\sigma}_{0}=|0\rangle\langle 0|+| 1\rangle\left\langle 1\left|, \quad \hat{\sigma}_{1}=\right| 0\right\rangle\langle 1|+| 1\rangle\left\langle 0\left|, \quad \hat{\sigma}_{2}=i\right| 1\right\rangle\langle 0|-i| 0\rangle\left\langle 1\left|, \quad \hat{\sigma}_{3}=\right| 0\right\rangle\langle 0|-| 1\rangle\langle 1| .
$$

The operator $\hat{\sigma}_{0 \ldots 0}$ is the identity, $\left(\hat{\sigma}_{i_{1} \ldots i_{n}}\right)^{2}=\hat{\sigma}_{0 \ldots 0}, \operatorname{tr} \hat{\sigma}_{0 \ldots 0}=2^{n}$, and $\operatorname{tr} \hat{\sigma}_{i_{1} \ldots i_{n}}=0$ if there is $i_{k} \neq 0$. The mutual unitary transformations of the Pauli basis operators give us another important relation: $\hat{\sigma}_{i_{1} \ldots i_{n}} \hat{\sigma}_{k_{1} \ldots k_{n}} \hat{\sigma}_{i_{1} \ldots i_{n}}= \pm \hat{\sigma}_{i_{1} \ldots i_{n}}$, where the plus sign takes place if and only if the number of triples $\left(i_{m} k_{m} i_{m}\right)_{m \in\{1, \ldots, n\}}$, satisfying the conditions $i_{m} \neq k_{m}, i_{m} \neq 0$, and $k_{m} \neq 0$, is even. It is also easy to see that the unitary transition matrix, transforming the standard basis $\left\{\left|i_{1} \ldots i_{n}\right\rangle\left\langle j_{1} \ldots j_{n}\right|\right\}$ into the Pauli basis, consists of only the elements $0, \pm 1$, and $\pm i$. In particular, the orthogonal projectors can be expressed as

$$
\left|i_{1} \ldots i_{n}\right\rangle\left\langle\left. i_{1} \ldots i_{n}\right|_{i_{1}, \ldots, i_{n} \in\{0,1\}}=\frac{1}{2^{n}} \sum_{k_{1}, \ldots, k_{n} \in\{0,3\}} \chi_{k_{1}}^{i_{1}} \cdots \chi_{k_{n}}^{i_{n}} \hat{\sigma}_{k_{1}} \otimes \ldots \otimes \hat{\sigma}_{k_{n}}, \quad \chi_{0}^{0}=\chi_{3}^{0}=\chi_{0}^{1}=1, \quad \chi_{3}^{1}=-1 .\right.
$$

The basis operators (1) span the real space $\mathcal{H}_{n}$ of Hermitian operators, and $2^{2 n}-1$ operators $\left\{i \hat{\sigma}_{i_{1} \ldots i_{n}}\right\}_{i_{1}, \ldots, i_{n} \in\{0,1,2,3\}}$, except $i \hat{\sigma}_{0 \ldots 0}$, span the Lie algebra of the Lie group $S U\left(2^{n}\right)$.

A quantum state (a density operator) is a positive semidefinite operator of the form

$$
\hat{\rho}=\frac{1}{2^{n}} \sum_{i_{1}, \ldots, i_{n} \in\{0,1,2,3\}} a_{i_{1} \ldots i_{n}} \hat{\sigma}_{i_{1} \ldots i_{n}},
$$

where

$$
a_{i_{1} \ldots i_{n}} \in \mathbb{R}, \quad a_{0 \ldots 0}=1, \quad\left|a_{i_{1} \ldots i_{n}}\right| \leqslant 1, \quad \frac{1}{2^{n}} \sum_{i_{1}, \ldots, i_{n} \in\{0,1,2,3\}}\left(a_{i_{1} \ldots i_{n}}\right)^{2} \leqslant 1 .
$$

The conditions (3) guarantee that $\hat{\rho}^{\dagger}=\hat{\rho}, \operatorname{tr} \hat{\rho}=1$, and $\operatorname{tr} \hat{\rho}^{2} \leq 1$. For our approach, it is important that all coefficients in the state (2) are real and each of them, except $a_{0 \ldots 0}$, is exactly the result of a local measurement, that is, a projection on one of the basis operators (1), $a_{i_{1} \ldots i_{n}}=\operatorname{tr}\left(\hat{\rho} \hat{\sigma}_{i_{1} \ldots i_{n}}\right)$. All the states make up a convex set $\mathcal{S}_{n}$ in the linear manifold $\left\{\hat{\sigma}_{0 \ldots 0}+\mathcal{H}_{n}\right\} \subset$ End $_{\mathbb{C}} \mathcal{V}_{n}$, while the pure states are placed on the boundary of $\mathcal{S}_{n}$ and make up a real submanifold of dimension $2^{n+1}-2$.

\section{Simulation of the state space by covariant expansions}

We will use the results of Ref. [11] where the problem of covariant expansions on manifolds is solved in the most general setting: one has only a given linear connection which is not assumed to be metric or torsion-free. Moreover, the coefficients of a covariant series can be defined on an arbitrary proper submanifold (not necessarily at a point); in our case, such a submanifold normally models an ancillary subsystem. The differential geometric structure of a four-dimensional manifold $M$, which will be used as a model of the state space of a $n$-qubit quantum system, is shown in Fig. 1. 


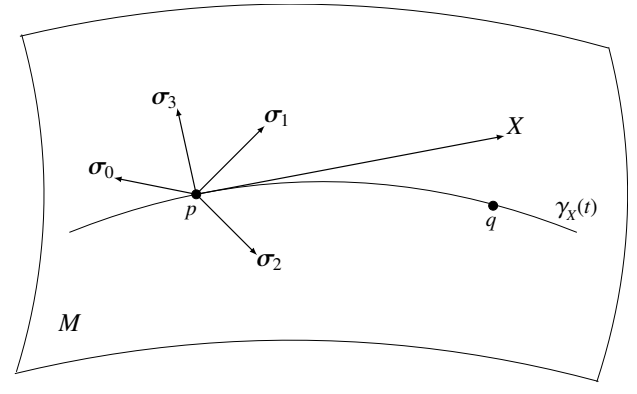

Figure 1. $M$ is a four-dimensional manifold with a linear connection $\nabla, p, q \in M, T_{p} M$ is the tangent space at $p$, and $X \in T_{p} M$. Let $\gamma_{X}(t)$ be a geodesic passing through $p$ in the direction of $X$ such that $\gamma_{X}(0)=p$ and $\gamma_{X}(1)=q$. The exponential map is a diffeomorphism of some open neighbourhood of $0 \in T_{p} M$ into an open neighbourhood of $p \in M$.

Let

$$
A_{n}=\sum_{i_{1}, \ldots, i_{n} \in\{0,1,2,3\}} a_{i_{1} \ldots i_{n}} \sigma_{i_{1}} \otimes \cdots \otimes \sigma_{i_{n}}
$$

be a smooth tensor field of rank $n$ on $M$. Here $\left\{\sigma_{0}, \sigma_{1}, \sigma_{2}, \sigma_{3}\right\}$ is an arbitrary (possibly noncoordinate) orthonormal basis of smooth vector fields on $M$. Each vector $X \in T_{p} M$ defines a geodesic $\gamma_{X}(t)$ in the direction of $X$ with the initial condition $\gamma_{X}(0)=p$. The exponential map, $\exp _{p}: X \mapsto \gamma_{X}(1)$, is a diffeomorphism of some (admissible) open neighbourhood of zero in $T_{p} M$ into an open neighbourhood of the point $p$. The inverse map $\exp _{p}^{-1}$ and a basis $\left\{\sigma_{0}, \sigma_{1}, \sigma_{2}, \sigma_{3}\right\}_{p} \subset T_{p} M$ define a coordinate map $\Phi$. If $X=X^{0} \sigma_{0}+X^{1} \sigma_{1}+X^{2} \sigma_{2}+X^{3} \sigma_{3}$ is a vector at a point $p \in M$, then $\Phi: p \mapsto(0,0,0,0)$ and $\Phi: q \mapsto\left(X^{0}, X^{1}, X^{2}, X^{3}\right)$, because these points are connected by a geodesic $\gamma_{X}(t)$ such that $p=\gamma_{X}(0)$ and $q=\gamma_{X}(1)$. The points $p \in M$ and $q \in M$ are associated, respectively, with an initial state and a final state of the quantum system; we will denote the components of the field $A_{n}$ at the points $p$ and $q$ as $a_{i_{1} \ldots i_{n}}$ and $\tilde{a}_{i_{1} \ldots i_{n}}$, respectively.

In what follows, we assume the summation convention for repeated Latin indices $i, j, k, l, m$ which run from 0 to 3 . For the sake of brevity, we will also deal with a linear connection with zero curvature and nonzero torsion. Let $\nabla_{\sigma_{i}} \sigma_{j}=\Gamma_{i j}^{k} \sigma_{k}$ be the covariant derivatives of basis vector fields, such that the components of the torsion are $T_{i j}^{k}=\Gamma_{i j}^{k}-\Gamma_{j i}^{k}$. In this case, the covariant expansions in normal coordinates $X^{0}, X^{1}, X^{2}, X^{3}$ take the form

$$
\tilde{a}_{i_{1} \ldots i_{n}}=\sum_{\sigma+|\mu| \geqslant 0} \frac{1}{\sigma !} X^{l_{1}} \ldots X^{l_{\sigma}} a_{k_{1} \ldots k_{r} ; l_{1} \ldots l_{\sigma}}^{l_{\left(\mu_{1}\right)}} \underset{i_{1}}{k_{1}} \ldots \underset{\left(\mu_{n}\right)}{k_{1} i_{r}} u_{i_{r}}^{k_{2}}, \quad \sigma, \mu_{1}, \ldots, \mu_{n} \geqslant 0,|\mu|=\mu_{1}+\ldots+\mu_{n}
$$

where the semicolon denotes a covariant derivative, and

$$
\underset{(0)}{u_{i}^{k}}=\delta_{i}^{k}, \quad \underset{(\mu)}{u_{i}^{k}}=\frac{1}{\mu+1} \sum_{\alpha=1}^{\mu} \frac{1}{(\alpha-1) !} X^{l_{1}} \ldots X^{l_{\alpha}}\left(T_{l_{1} m ; l_{2} \ldots l_{\alpha}}^{k}\right)_{p(\mu-\alpha)^{i}}^{u} \underset{m}{m}(\mu 1) .
$$

There are many ways to use these series. In the general case, it can be shown that an arbitrary state admits a high-fidelity approximation by the expansions (5) in which all components in (4) are constant or linear functions (in the basis $\left\{\sigma_{i}\right\}$ ), and the terms with covariant derivatives of curvature and torsion as well as of the field $A_{n}$ vanish. In the latter case the computations become much more complicated and extended, but this approach guarantees that the state transformation will be unitary for all $t \in[0,1]$. It should be stressed that one has to compute the components in the basis $\left\{\sigma_{i}\right\}$, not in the coordinate basis of normal coordinates; a computation algorithm was proposed in [12].

Note that in a system of $n$ qubits, one has $2^{n}-1$ partitions into two complementary pairs of subsystems, and for each pair the von Neumann entropy can be easily computed in terms of the coefficients $a_{i_{1} \ldots i_{n}}$. Moreover, even in cases where we do not know the desired final state, this method allows us to model any system with specified permissible entropies of its subsystems. 


\section{An example: $\hat{\rho}_{3}^{0} \rightarrow \mathrm{GHZ}_{3}$}

Without loss of generality, one can take $\hat{\rho}_{3}^{0}=|000\rangle\langle 000|$ as the initial state of a 3-qubit system. In the Pauli basis, $\hat{\rho}_{3}^{0}=\frac{1}{8}\left(\hat{\sigma}_{000}+\hat{\sigma}_{003}+\hat{\sigma}_{030}+\hat{\sigma}_{300}+\hat{\sigma}_{033}+\hat{\sigma}_{303}+\hat{\sigma}_{330}+\hat{\sigma}_{333}\right)$ and $\mathrm{GHZ}_{3}=$ $\frac{1}{8}\left(\hat{\sigma}_{000}+\hat{\sigma}_{033}+\hat{\sigma}_{303}+\hat{\sigma}_{330}+\hat{\sigma}_{111}-\hat{\sigma}_{122}-\hat{\sigma}_{212}-\hat{\sigma}_{221}\right)$. For $n=3$ we have (third-order terms are needed but omitted because of their lengthy form)

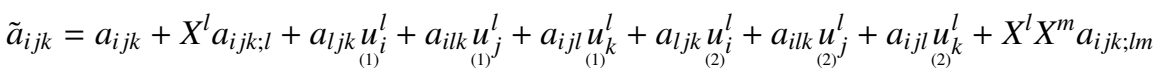

$$
\begin{aligned}
& +X^{m} a_{l j k ; m} \underset{(1)}{u_{i}^{l}}+X^{m} a_{i l k ; m} \underset{(1)}{u_{j}^{l}}+X^{m} a_{i j l ; m} \underset{(1)}{u_{(1)}^{l}}+a_{l m k} \underset{(1)}{u_{i}^{l}} u_{j}^{m}+a_{l j m} \underset{(1)}{u_{(1)}^{l}} u_{k}^{l} u_{k}^{m}+a_{i l m} \underset{(1)}{u_{j}^{l}} u_{(1)}^{u_{k}^{m}}+O\left(\|X\|^{3}\right) .
\end{aligned}
$$

For any Lie group, there exist canonical connections defined in the basis of left-invariant fields. If one defines $\nabla_{\sigma_{i}} \sigma_{j}=\left[\sigma_{i}, \sigma_{j}\right]$, such that $R=0$ and $T\left(\sigma_{i}, \sigma_{j}\right)=\left[\sigma_{i}, \sigma_{j}\right]$, then summands with covariant derivatives of the torsion will be zero everywhere. If in addition the components (in the basis $\left\{\sigma_{i}\right\}$ ) of the field $A_{3}$ are zero or linear functions, then their covariant derivatives at the point $p$ are equal to zero or constant, and all the functions $\underset{(\mu)^{k}}{u_{i}^{i}}(\mu \geq 2)$ can be expressed in terms of $\underset{(1)^{k}}{u_{i}^{i}}=(1 / 2) X^{l} T_{l i}^{k}$.

For the transformation $\hat{\rho}_{3}^{0} \rightarrow \mathrm{GHZ}_{3}$, a possible approach is to use the algebra Lie of the group $S L(2, \mathbb{R}) \times \mathbb{R},\left(\left[\sigma_{0}, \sigma_{1}\right]=\sigma_{1},\left[\sigma_{0}, \sigma_{2}\right]=-\sigma_{2},\left[\sigma_{1}, \sigma_{2}\right]=\sigma_{0},\left[\sigma_{3}, \sigma_{\alpha}\right]=0, \alpha=0,1,2\right)$, and then to set $a_{000}=a_{033}=1, a_{001}=X^{2}, a_{002}=-X^{1}, a_{011}=a_{012}=a_{112}=a_{022}=a_{122}=a_{013}=a_{023}=0$, $a_{111}=-X^{2}, a_{222}=-3 X^{1} / 8, a_{003}=a_{333}=1-X^{1}$ (plus the relations with permuted indices), $\left(X^{0}, X^{1}, X^{2}, X^{3}\right)_{q}=\left(0,1,-2 / 3^{1 / 3}, 0\right)$. The nonzero torsion components and the transformation matrices are $T_{01}^{1}=-T_{02}^{2}=T_{12}^{0}=1, \quad \underset{(1)}{u_{0}^{2}}=-\underset{(1)}{u_{1}^{0}}=\frac{1}{2} X^{1}, \underset{(1)}{u_{2}^{0}}=-\underset{(1)}{u_{0}^{1}}=\frac{1}{2} X^{2}, \underset{(2)}{u_{2}^{1}}=-\underset{(2)}{u_{1}^{2}}=-\frac{1}{4} X^{1} X^{2}$.

\section{Conclusion}

We presented a new method for modelling tensor network states in complex quantum systems with a large number of qubits. The method requires $m \leq n^{3}$ (the number of nonzero connection coefficients) varying parameters for a deterministic simulation of an arbitrary state on a classical computer; in the example above, $n=3$ and $m=2$. In the general case, it is hard to obtain a good estimation of the fidelity of the approximation and the complexity of the computations, but it is clear that $n$-th order terms in (5) should be taken into account.

\section{References}

[1] I. Bengtsson, K. Zyczkowski, Geometry of Quantum States: An Introduction to Quantum Entanglement (Cambridge University Press, Cambridge, 2006)

[2] Y. Ling, Y. Liu, Z.-Y. Xian, Y. Xiao, Phys. Rev. D 99, 026008 (2019)

[3] S.S. Jahromi, R. Orus, Phys. Rev. D 99, 195105 (2019)

[4] G. Evenbly, G. Vidal, J. Stat. Phys. D 145, 891 (2011)

[5] L.H. Kauffman, E. Mehrotra, Quant. Inf. Process 18, 3, 76 (2019)

[6] E. Bianchi, P. Dona, I. Vilensky, Phys. Rev. D 99, 086013 (2019)

[7] M. Van Raamsdonk, Int. J. Mod. Phys. D 19, 2429 (2010)

[8] P. Mukhopadhyay, Reviews in Math. Phys. 26, 1, 1350019 (2013)

[9] L. Brewin, Class. Quantum Grav. 26, 175017 (2009)

[10] U. Müller, C. Schubert, A.E.M. van de Ven, Gen. Relativ. Grav. 1, 1759-1765 (1999)

[11] A.N. Tsirulev, Theor. Math. Phys. 102, 3, 245 (1995)

[12] I.M. Potashov, A.N. Tsirulev, EPJ Web of Conf. 173, 03021 (2018) 